\title{
Estudios toponímicos en Venezuela. Una revisión a favor de un reconocimiento de su valor integral
}

\author{
Toponimic studies in Venezuela. A review in favor of a recognition \\ of its integral value
}

- ANERKIS CANACHE

canache.anerkis@gmail.com

Código ORCID: 0000-0001-8708-1883

Universidad Pedagógica Experimental Libertador, Venezuela

Artículo recibido en octubre 2020 / Arbitrado en noviembre 2020 / Publicado en enero 2021

Resumen El objetivo de la presente investigación fue determinar y sistematizar las investigaciones toponímicas realizadas en Venezuela, con el fin de presentar una aproximación al estado de conocimiento y un reconocimiento de su valor integral por su relación con diversos campos del saber. La investigación fue de enfoque cualitativo y diseño documental. Para ello se realizó un arqueo bibliográfico de donde se obtuvieron dieciséis trabajos de Venezuela. Se utilizaron las técnicas del fichaje y del resumen. Los instrumentos fueron la ficha y el cuaderno de campo. Los criterios utilizados para el análisis fueron: tendencia de los estudios, método en la investigación toponímica, origen del nombre, la obra toponímica y otros datos de interés. Los resultados arrojaron que las investigaciones presentaron tendencias inter $\mathrm{y} / \mathrm{o}$ transdisciplinarias, aunque predominó el orden lingüístico. Esto evidencia que en estos estudios están inmersas diversas áreas del saber lo que les otorga un valor integral.

Palabras clave: Onomástica; topónimo; toponimia; Venezuela

\begin{abstract}
The objective of this research was to determine and systematize the toponymic research carried out in Venezuela, in order to present an approximation to the state of knowledge and recognition of its integral value due to its relationship with various fields of knowledge. The research was of qualitative approach and documentary design. For this, a bibliographic survey was carried out from where sixteen works were obtained from Venezuela. The signing and summary techniques were used. The instruments were the record and the field notebook. The criteria used for the analysis were: trend of studies, method of toponymic research, origin of name, toponymic work and other data of interest. The results showed that the research presented inter and / or transdisciplinary tendencies, although the linguistic order prevailed. This shows that various areas of knowledge are immersed in these studies, which gives them an integral value.

Keywords: onomastics; place name; toponymy; Venezuela
\end{abstract}

Keywords: Onomastics; place name; toponymy; Venezuela 


\section{INTRODUCCIÓN}

La dinámica humana involucra una extensa variedad de elementos que otorga al individuo, particularmente, mecanismos por medio de los que se van perfeccionando las relaciones sociales, entre esos elementos se cuenta la lengua.

La lengua tiene un carácter social, cuyos dispositivos propician un complejo entramado lingüístico, en torno al cual giran diversos componentes (fonológico, léxico, semántico, morfosintáctico y pragmático) que, a su vez, tanto la lingüística como sus disciplinas se encargarán de explicar.

Cada hablante, al hacer uso de la lengua, crea y recrea el entorno en el cual se encuentra inmerso, de esto pueden dar cuenta la lexicografía y la lexicología como disciplinas lingüísticas. Designar nombres a los lugares también es una muestra del acto comunicativo, convirtiéndose en un indicador de la necesidad de los seres humanos por reconocerse dentro de un espacio e identificarse, así mismo, con dicho contexto.

Con la finalidad de indagar acerca de los nombres propios surge la onomástica, a objeto de especificar el estudio de los nombres como un aspecto perteneciente a la lingüística. Dentro de la onomástica, la toponimia se encarga del estudio de los nombres de lugar en cuanto a su origen, características y evolución.

En este sentido, la propuesta del presente estudio se orientó a determinar y sistematizar las investigaciones toponímicas que se han realizado en Venezuela; con lo que se pretende, a grandes rasgos, una aproximación al estado de conocimiento y un reconocimiento del valor integral que posee este tipo de estudios.

Para problematizar se tuvo que empezar por la complejidad del acto de nombrar. Designar un nombre es un proceso que implica considerar algunas características y/o condiciones específicas del objeto, persona, lugar, entre otros. Quien inventa o diseña un objeto, por ejemplo, además de crear todas las funciones que este va a cumplir, le confiere un nombre que no solo va a permitir identificar al objeto sino que también aportará indicios de lo que es en sustancia (Arteaga y Cova, 2009).

Cuando una mujer está esperando un bebé comienza una minuciosa búsqueda del nombre que ha de honrar la existencia de ese nuevo ser humano que está por nacer; tomando en cuenta los rasgos familiares, la historia del posible nombre o las distintas combinaciones que pudieran darse entre los nombres del núcleo familiar.

Hay nombres que para algunos resultan interesantes; estos son los nombres de lugares. Cuando se visita o se conoce un lugar nuevo es posible que, por indagar un poco más acerca de este, llame la atención saber por qué tiene tal o cuál nombre, a qué o a quién se deberá esa designación, si siempre se habrá llamado de esa manera y un sin número de preguntas más.

Primeramente, es importante destacar que al nombre de lugar se le conoce como topónimo. También conviene saber que existen distintos elementos que intervienen en su designación, pues en torno a su nominación giran factores de diversa índole como las características geográficas, 
culturales, botánicas, sociales, solo por mencionar algunas.

De acuerdo con lo definido en el Diccionario de lingüística (1986) la toponimia es la disciplina que se encarga del estudio de los nombres de lugar en cuanto a su origen, evolución y significado. A la luz de esta disciplina, son variadas las razones por las que se estudian estos nombres, así como también son distintas las disciplinas en las que la toponimia se apoya para dar respuesta a las necesidades del investigador. Cada estudio toponímico es una aproximación que permite la reconstrucción del nombre en cuestión, pues de manera integral, se involucran en estos estudios aspectos históricos, lingüísticos, antropológicos, geográficos, sociales, culturales, económicos, entre otros, que en mayor o menor medida contribuyen con el origen, evolución y existencia del nombre.

Al analizar un topónimo se inicia un largo recorrido por diversas situaciones que ponen en perspectiva el origen del nombre que ocupa el estudio. Por ejemplo: culturalmente, a cuál grupo representa; lexicográficamente, cuál es su étimo, su origen; lingüísticamente, a cuál lengua pertenece; geográficamente, si este representa una característica del terreno; etc. En forma general, su origen puede ser indígena, hispano $\mathrm{y} / \mathrm{o}$ africano $\mathrm{y}$, de una manera más particular. Páez (2001) menciona una clasificación que va desde orónimos (nombres relativos a formación montañosa); hidrónimos (nombres relativos a corrientes de agua); fitónimos (nombres relativos a plantas); hasta zoónimos (nombres relativos a animales).
Conocer todos estos datos involucra minuciosos procesos de búsqueda $\mathrm{y}$ sistematización de la información, a partir de la presente investigación se pretende recoger varios de los trabajos toponímicos que se han desarrollado en el país, en cuanto al corpus seleccionado, la metodología empleada, los resultados obtenidos y la presentación de la información, con el objeto de destacar la labor realizada por sus autores.

Es importante hacer ver que al respecto no se han encontrado trabajos actualizados que presenten información sistematizada de las investigaciones toponímicas en el país; tarea que precisa ser llevada a cabo con el fin de que se generen respuestas a las necesidades de investigación en esta rama del conocimiento. Necesidades que van desde hallar un registro de los estudios toponímicos desarrollados hasta el presente, que pueda convertirse en una herramienta de gran utilidad, pues reduciría el tiempo invertido en la búsqueda de esta información por separado. Incluso obtener, por medio de esa misma herramienta, los datos que permitan establecer las valoraciones que cada investigador ha de ajustar a las exigencias de su estudio.

Debido a la lenta o postergada labor de sistematización es impreciso el registro de los trabajos toponímicos realizados en el país, lo que ha traído como consecuencia que: a) se desconozcan las investigaciones toponímicas hechas, (b) se ignoren las tendencias en las investigaciones, (c) no se establezcan parámetros para su clasificación por áreas de conocimiento, y (d) se 
obstaculice la valoración y vigencia de dichos estudios y, por ende, su divulgación.

Los estudios toponímicos se amparan en la versatilidad de las investigaciones lingüísticas, debido a que estas ofrecen explicaciones de la lengua y de sus hablantes, por lo que se considera necesaria la sistematización de estos estudios para que exista una referencia que proporcione datos primarios que orienten investigaciones similares e inéditas.

\section{MÉTODO}

Cuando se emprende una investigación se realiza una serie de procedimientos inherentes a actividades de esta índole. Generalmente se establece una especie de protocolo, por medio del cual es organizado el desarrollo del estudio. Conforme a lo dicho, se presenta a continuación el abordaje metodológico desarrollado para este estudio.

La toponimia en Venezuela es un trabajo que coincide, en cuanto al tipo de investigación, con los criterios cualitativos; para precisarlo es oportuno citar a Rodríguez, Gil y García (1996):

Los investigadores cualitativos estudian la realidad en su contexto natural, tal como sucede, intentando sacar sentido de, o interpretar, los fenómenos de acuerdo con los significados que tienen para las personas implicadas. La investigación cualitativa implica la utilización y recogida de una gran variedad de materiales que describen la rutina y las situaciones problemáticas y los significados en la vida de las personas. (p. 72)
En efecto, esta investigación descansa sobre lo descrito por Rodríguez, Gil y García (1996), pues se recogieron y analizaron datos que refieren una realidad, en este caso, relativos a algunos de los estudios toponímicos que se han hecho en el país, considerando para ello no solo el valor que tiene para quien lo elabora, sino también en diferentes áreas del conocimiento.

Dadas sus características y de acuerdo con Alfonzo (1994) esta es una investigación documental debido a que es aplicado un proceso sistemático de búsqueda, recolección, clasificación, análisis e interpretación de la información encontrada.

De igual modo, en el Manual de Trabajos de Grado de Especialización y Maestría y Tesis Doctorales (UPEL, 2016) se enfatiza que a través de la investigación documental se abordan "problemas con el propósito de ampliar y profundizar el conocimiento de su naturaleza, con apoyo, principalmente, en trabajos previos, información y datos divulgados por medios impresos, audiovisuales o electrónicos" (p. 20).

Cázares, Christen, Jaramillo, Villaseñor y Zamudio (2000) denominan a la investigación documental como aquella que:

... depende fundamentalmente de la información que se recoge o consulta en documentos, entendiéndose este término, en sentido amplio, como todo material de índole permanente, es decir, al que se puede acudir como fuente o referencia en cualquier momento o lugar, sin que se altere su naturaleza o sentido, para que aporte 
información o rinda cuentas de una realidad o acontecimiento. (p. 8)

Sin duda, esta es una investigación documental, puesto que las fuentes de las que se ha extraído la información que compone este trabajo gozan de un estado de permanencia, esto se refiere a que puede ser hallada y consultada cada vez que el investigador así lo desee y lo requiera, debido a que su presentación está impresa o digitalizada.

Es importante enfatizar que los trabajos a la luz de esta modalidad pueden ser, de acuerdo con Cázares et al. (2000) "Estudios de investigación histórica, literaria, geográfica, matemática $u$ otros propios de las especialidades de los subprogramas" (p. 20).

En este sentido, aunque se apoya el señalamiento del autor, el presente trabajo de La Toponimia en Venezuela no se circunscribió a un área específica, de igual modo se evidencia el aporte inter y transdisciplinario en la conformación de la obra toponímica.

Es preciso describir la forma en que se obtuvo, organizó y procesó la información contenida en este trabajo.

Efectivamente, se inició con el arqueo bibliográfico, empleado para sincerar la utilidad de lo recabado, es decir, lo que serviría y lo que no en beneficio del desarrollo de la investigación.

Como contribución intelectual, se tomaron en cuenta los procesos cognitivos básicos con el objeto de sacar el mayor provecho a las técnicas e instrumentos seleccionados $y$, sobre todo, para facilitar la tarea de comprensión e interpretación de la información.
En apoyo a la dimensión organizativa en la que ya se encontraba inmerso el trabajo, se recurrió a las técnicas del fichaje y el resumen. El fichaje, para Puente (s.f), "es una técnica auxiliar de todas las demás técnicas empleada en investigación científica; consiste en registrar los datos que se van obteniendo" (s.p), durante el proceso de recolección y selección del material bibliográfico.

La utilización del fichaje permitió ponderar el hallazgo que se iba produciendo durante cada visita a los centros de documentación $\mathrm{y} / \mathrm{o}$ en las revisiones electrónicas. Carrera, Vásquez y Díaz (2006) le atribuyen distintas ventajas a esta técnica, entre ellas la de permitir "una rápida y cómoda clasificación de los materiales" ( $p$. 171). Efectivamente esta facilitó y agilizó la organización del insumo documental, electrónico y más.

Con respecto al resumen, Alfonzo (1994) lo define como "la exposición condensada de un escrito en el cual se refleja fielmente las ideas expresadas en el texto original" ( $p$. 117).

El resumen facilitó la extracción de los datos que coincidían con los objetivos propuestos para esta investigación, se admitió con esta técnica concebir un conjunto de criterios en los que, posteriormente, se fundamentarían las valoraciones y conclusiones planteadas para este estudio.

En otras palabras, por medio de la técnica del resumen fue posible exponer las ideas de los autores que concurren en la presente revisión, bien para mostrar la esencia de sus trabajos, como para precisar las respuestas que satisfagan las expectativas de la autora de este trabajo documental. 
Para aplicar las técnicas seleccionadas, se precisó de algunos instrumentos tales como la ficha y el cuaderno de campo.

Puente (s.f) describe a la ficha como instrumentos que "debidamente elaboradas y ordenadas contienen la mayor parte de la información que se recopila en una investigación por lo cual constituye un valioso auxiliar en esa tarea" ( $\mathrm{s} / \mathrm{p}$ ).

Con la ficha se ejecutó el registro de todas y cada una de las pistas, por así decirlo, de los trabajos que son reseñados en la presente investigación. En las fichas se hacía referencia a la información esencial de las obras que eran encontradas, para luego llegar a ellas de una manera más directa y precisa.

Por su parte, el cuaderno de campo representa, al igual que la ficha, un instrumento con un valioso contenido. Taylor y Bogdan (1987) resaltan algunas condiciones a las que se sujetan los apuntes en esta herramienta; dichas condiciones dependen, en gran medida, de las necesidades y criterio del investigador, pues éste será quien determine el cuándo, cómo, qué y para qué de lo registrado en él.

El cuaderno de campo sirvió, entonces, para registrar todo cuanto se pensó que sería de utilidad en la conformación de este trabajo, desde palabras clave hasta ideas que se fueron constituyendo en apartados.

La selección y utilización de las técnicas sirvieron para ir cumpliendo con los objetivos trazados en la investigación. A continuación el procedimiento es enumerado en dos etapas.

\section{Etapa. Recolección y análisis de la información}

Esta etapa se desarrolló en dos fases:

\section{Fase}

Después de haber delimitado el tema de estudio, se comenzó a recabar la información respectiva, procurando llegar a una aproximación del estado real del tema seleccionado. Se realizaron visitas a varios centros de documentación, entre ellos la biblioteca del Instituto Pedagógico de Miranda José Manuel Siso Martínez, la del Instituto Pedagógico de Caracas, la Biblioteca Nacional. También fueron revisados sitios en Internet, así como textos personales, de los que pudieron extraerse datos útiles que alimentaron la investigación desarrollada. En la I etapa el uso de la técnica del fichaje permitió la selección de la información obtenida durante las visitas y revisiones.

\section{Fase}

Concluida la fase de recolección, el siguiente paso fue el de sincerar y analizar el material ya fichado en atención con los objetivos del estudio.

Fue indispensable determinar, a través del resumen de los estudios toponímicos en Venezuela encontrados, la siguiente información: el objeto de la investigación, la metodología empleada, los resultados obtenidos y la manera como fueron presentados; aspectos que se retomaron en la segunda etapa de esta investigación, para identificar las pistas a partir de las que se hizo el contraste entre los diferentes trabajos hallados. 


\section{Etapa. Sistematización de los registros}

En esta última etapa se establecieron los mecanismos para la categorización de los trabajos registrados, se partió de la información puntualizada en la segunda fase, por ejemplo, en cuanto a la tendencia del estudio, las disciplinas en las que se apoyan los estudios toponímicos en Venezuela, la metodología empleada, los resultados alcanzados y la forma de presentar la investigación, entre otros.

La idea era comparar la información para construir una matriz que permitiera apreciar las coincidencias y particularidades en los estudios reseñados. Gracias a ello, en cada uno de los trabajos se dejaron ver interesantísimas muestras inter y transdisciplinarias contenidas en la toponimia venezolana y con lo que, finalmente, se concretan los objetivos propuestos en esta investigación.

\section{RESULTADOS}

Los resultados analizados de los trabajos toponímicos se organizaron en orden decreciente (desde el más reciente hasta el más antiguo), tarea propuesta como primer objetivo del presente trabajo; posteriormente la información se puntualizó considerando como criterios la tendencia de los estudios, el método en la investigación toponímica, el origen del nombre, la obra toponímica y otros datos de interés.

\section{Etapa. Recolección y análisis de la información}

Es importante recordar que los estudios toponímicos encontrados, específicamente dieciséis desarrollados en Venezuela, fueron contrastados con el modelo metodológico y procedimental propuesto por Salazar (1990) y que se reflejan a través de sus distintas obras. Salazar (1990) toma en cuenta, de una manera meticulosa, aspectos que le otorgan contundencia al estudio de los nombres de lugar, no obstante, es necesario resaltar que cada investigación, en sí, suma elementos de utilidad para este tipo de trabajo.

\section{Etapa. Sistematización de los registros}

Las categorías que se obtuvieron, luego de culminada la I Etapa, se describen a continuación:

\section{Tendencias en los estudios toponímicos en Venezuela}

El nombre de un lugar posee características específicas que, como las dos caras de una misma moneda, por un lado lo hace único y por el otro lo agrupa entre semejantes; esta es una de las labores emprendidas por Salazar (1990) por medio de cada uno de sus trabajos, es decir, describir la peculiaridad del topónimo, así como, su relación con elementos de diversa índole.

Por ejemplo, él establece una clasificación del topónimo en función de su importancia: a) Cartográfica, b) jurídica, c) geográfica, d) histórica, e) folklórica, f) político-administrativa, g) lingüística, h) patrimonial, i) vial, y j) social. Atendiendo a esta clasificación, es oportuno añadir una undécima categoría denominada biológica. 
En este caso, la biológica estaría representada por aquellos estudios en los que se valora el reflejo de la flora y/o la fauna en los topónimos. En ellos se describe el origen del nombre del lugar, atendiendo a su particularidad, aparición, registro y correspondencia con el elemento de la flora o fauna respectivo.

Los estudios toponímicos seleccionados para esta investigación, de acuerdo con su preponderancia, se sitúan en el orden: a) cartográfico, b) histórico, c) folklórico, d) lingüístico, e) patrimonial, y f) biológico, respectivamente.

Entre los de orden cartográfico se ubican todos aquellos trabajos cuyo objeto de estudio se fundamenta en los insumos provenientes de cartas de navegación, mapas $y$ atlas antiguos $u$ otros documentos de esta índole, debido a que en ellos la búsqueda se orienta hacia los primeros registros del nombre de un lugar. Por apoyarse en la pauta antes descrita, se circunscriben al orden cartográfico La Toponimia en Venezuela (1985) y La Toponimia Venezolana en las Fuentes Cartográficas del Archivo General de Indias (1983), ambas obras pertenecientes a Salazar.

Las investigaciones de orden patrimonial se caracterizan por su condición de resistencia al cambio, impuesta por el tiempo, que poseen algunos nombres de lugar en los que se ven reflejados el sentido de pertenencia, la identidad y la preservación que los convierten en patrimonio cultural de una región, de un país.

En este sentido, los nombres de lugar guardan valiosos datos que forman parte del acervo cultural e histórico del lugar al cual pertenecen. Describir el origen de un topónimo implica develar información con un alto valor patrio. Esta es la razón por la que la Toponimia Carabobeña (1997) y la Toponimia del Delta del Orinoco (1990) de Idler y Salazar, respectivamente, se enmarcan en los estudios de este orden.

Ajustados al orden lingüístico se encuentra la Toponimia Indígena del Municipio Vargas (2005) de Acevedo, El Origen del Nombre de Venezuela es Indígena, y quiere decir Agua Grande de Hernández (s.f), el Diccionario Toponímico del Estado Carabobo (1996) de Esté, Esteller y Rondón el Diccionario Toponímico de Venezuela (1992) de Chiossone y la Toponimia Indígena de Aragua (1990) de Botello.

Estos son estudios que descansan en las dimensiones etimológicas y léxico-semántica de los nombres de lugar, profundizando en la o las palabras que componen el nombre para ofrecer una descripción y explicación de su procedencia, significado y evolución. Esto se debe a que la palabra (nombre) que se designa a algún lugar es susceptible de ser analizada, esencialmente, desde dichas dimensiones, por tanto, entra o se ubica dentro de la categoría lingüística.

Casale (1997), autor de La Fitotoponimia de los pueblos de Venezuela, y Mata (1997), autor de Toponimia de Pueblos Neoespartanos, por medio de sus obras procuran dar razón de aquellos nombres de plantas designados a lugares, así como de sus variaciones y ortografía. Esta tendencia se desarrolla bajo el orden biológico, campo en el que se apoya el investigador para dar respuesta, en este caso, a lo relacionado con la vegetación y su correspondencia con los 
nombres de lugar, valorando el reflejo de la flora y/o la fauna al describir el origen del nombre del lugar, en atención a las características, aparición y registro de los elementos naturales.

En esta revisión también se encontraron investigaciones de orden histórico, que se corresponden con aquellas en las que se intenta establecer una especie de recorrido a lo largo del tiempo a partir de los registros más antiguos que puedan existir del o los nombres objeto de estudio. El carácter de la fuente puede ser variado, lo imprescindible es la antigüedad del registro. Buenas y malas palabras de Rosenblat (2004) y Toponimia de Pueblos Neoespartanos de Mata (1997) coinciden con el patrón temporal brindado a los estudios de este orden.

En la misma investigación realizada por Mata (1997), Toponimia de Pueblos Neoespartanos, se encontraron evidencias de las creencias, costumbres y tradiciones manifestadas en los nombres de algunos lugares del estado Nueva Esparta, lo que puede traducirse en que por medio de esos nombres es posible la percepción de muchas de las prácticas adoptadas por un grupo de habitantes de determinada localidad.

La tendencia se orienta en describir la presencia de las manifestaciones culturales o expresiones de la idiosincrasia popular en los nombres de lugar o viceversa, lo que permite ubicar a este tipo de estudio dentro del orden folklórico.

\section{El método en la investigación toponímica}

Indagar en el origen de los nombres de lugar implica valerse de los más variados y remotos registros que puedan contribuir a la aproximación de su nacimiento.

En los trabajos que aquí se presentan, el procedimiento se orienta, en su mayoría, a la revisión documental que va desde mapas, cartas de navegación, documentos históricos, otras investigaciones y material electrónico.

La idea es constatar los datos de aparición del o los nombres objeto de estudio en la mayor cantidad de fuentes posibles; mientras que, el origen de la información hallada va a estar determinado por la orientación y necesidades de la investigación, tomando como referencia la clasificación propuesta por Salazar (1990).

\section{El origen del nombre de lugar}

El resultado de cada uno de los trabajos toponímicos responde a la importancia del topónimo $y$, por ende, al valor otorgado por el investigador; por lo tanto, indagar en la procedencia de los nombres de lugar dependerá de aspectos tan diversos como, por ejemplo: lo registrado en los más antiguos mapas de Venezuela y sus alrededores; el sentido de pertenencia y conservación del patrimonio regional expresado en la denominación de los lugares; el significado de las designaciones, atendiendo a las dimensiones etimológica y léxico-semántica; nombres cuya descripción y explicación puede darse desde la biología; la historia como objeto de la toponimia y la toponimia como objeto de la historia; así como, el reflejo de la cultura, la identidad y el folklore en los topónimos.

En suma, el enigma del origen del nombre viene dado por características distintas que favorecen la asignación del 
nombre a un lugar, dichas particularidades pueden producirse, por citar algunas razones, por un hecho o personaje histórico, por una tradición, por el sentido de pertenencia e identidad, por una comunidad de hablantes y su lengua.

\section{La naturaleza de la obra toponímica}

El producto del trabajo toponímico es variado, pues en él se expone la perspectiva del investigador en cuanto al origen del nombre de un lugar; gracias a ello la información es presentada de diferentes formas, entre las que se pueden encontrar textos, glosarios, diccionarios, compilaciones, artículos en internet y otros semejantes.

La presentación de la información dependerá del impacto y uso que el autor o autores deseen causar en los destinatarios de sus trabajos. No obstante, más que la intención con la que fue creada la obra, el usuario es quien va a determinar su valor y utilidad; así mismo, aunque no haya sido el fin para el que fue hecho el material, inevitablemente se convertirá en referencia obligatoria, en el buen sentido de la palabra, para el investigador toponímico.

\section{Otros datos de interés}

Para indagar acerca de los nombres de lugar no es necesario ser practicante de una disciplina en particular, es decir, el área de dominio del investigador no representa una limitante pues los estudios de este tipo son tan versátiles que permiten que quien investigue se apropie de todos los recursos disponibles en otros campos del conocimiento para satisfacer sus pretensiones.
De los estudios hallados y reseñados en el presente trabajo se encontró que las profesiones de sus autores son de áreas diversas, como: la antropología, la historia, las humanidades, la biología, la filología, la lingüística, entre otras.

Esta condición apoya la orientación de los estudios toponímicos, debido a que cada investigador inclinará su búsqueda hacia su área de desempeño, lo que dará como resultado la variedad de trabajos como los que aquí han sido presentados.

A pesar de que el área del investigador marcará la tendencia del estudio, es de suma importancia destacar la inter y la transdisciplinariedad de los trabajos toponímicos que ha podido evidenciarse a través de la confluencia de distintas áreas del conocimiento en una misma investigación, cuya contribución se ve materializada en la satisfacción de un solo interés que no es otro que exponer el origen del nombre de lugar.

\section{CONCLUSIONES}

El proceso de sistematización proporciona información relevante y puntualizada de las investigaciones toponímicas que se han realizado en el país. Es una herramienta que facilita la organización de los datos encontrados para su posterior acceso a ellos. Se pudo aprovechar la sistematización debido al aporte que, en este sentido, brinda Salazar (1990) en cada una de sus obras. La sistematización es considerada como un método para abordar estudios toponímicos.

Por otra parte, determinar el número de estudios toponímicos que se han hecho en el país es una labor que requiere de una 
cuantiosa dedicación temporal, por lo que se piensa que debido a la falta de inversión de tiempo no exista un registro franco $y$ sistematizado de los estudios toponímicos realizados en Venezuela. La cantidad de estudios reportados para esta revisión estuvo representada por veintiún obras, de las cuales cinco pertenecen a trabajos realizados en otros países y dieciséis, que fueron seleccionados para el análisis de la presente investigación, por haber sido desarrollados en el país.

El corpus toponímico de los trabajos reseñados en esta investigación, de acuerdo con la clasificación brindada por Salazar (1990), en función de su importancia, fueron de orden: a) cartográfico, b) patrimonial, c) lingüístico, d) biológico, e) histórico, y f) folklórico. Por lo tanto, la importancia, en cuanto a la tendencia del estudio realizado, dependió del resultado de la investigación toponímica, porque con ello se pudo dar respuesta al origen del nombre de lugar desde cada una de esas perspectivas.

Las investigaciones que se registraron en el presente trabajo presentaron tendencias variadas, en algunos casos específicas, en otros inter $y / o$ transdisciplinarias; pero uno de los aspectos más resaltantes, en este sentido, fue la predominancia de los estudios de orden lingüístico.

El método empleado para desarrollar las investigaciones toponímicas consiste, básicamente, en revisar los registros que alrededor del objeto de estudio se encuentren. Lo importante de la revisión radica en hallar, en la medida de lo posible, los registros más antiguos, puesto que estos le darán mayor solidez, veracidad y contundencia a los datos y las apreciaciones que de ellos se deriven.

La inter y/o transdisciplinariedad es una de las particularidades en estos estudios, la cual permite buscar respuesta del nombre de lugar desde cualquier perspectiva $y$, al mismo tiempo, se sirve de más de un área del conocimiento para satisfacer ampliamente las necesidades del investigador, lo que, a su vez, proporciona un amplio panorama del objeto de estudio.

\section{REFERENCIAS}

Acevedo, J. (2005). Toponimia indígena del municipio Vargas. Vargas: Fondo Editorial Urimare

Alfonzo, I. (1994). Técnicas de investigación bibliográfica. Caracas: Contexto-Editores

Arteaga, M. y Cova, Y. (2009). El nombre propio en Venezuela un estudio de onomástica descriptiva. Caracas: Universidad Pedagógica Experimental Libertador

Botello, O. (1990). Toponimia indígena de Aragua. Aragua: Publicaciones del Concejo del Municipio Girardot. Oficina del Cronista de la Ciudad. Villa de Cura: Editorial Miranda

Carrera, L., Vásquez, M. y Díaz, M. (2006). Técnicas de redacción e investigación documental. Caracas: Editorial Panapo

Casale, I. (1997). La fitotoponimia de los pueblos de Venezuela. Caracas: Ediciones de la Comisión Nacional de Nombres Geográficos

Cázares, L., Christen, M., Jaramillo, E., Villaseñor, L. y Zamudio, L. (2000). Técnicas actuales en la investigación documental. Ciudad de México: Trillas

Chiossone, T. (1992). Diccionario toponímico de Venezuela. Caracas: Biblioteca de Autores y Temas Tachirenses 
Diccionario de lingüística. (1986). Madrid: Anaya

Esté, M., Esteller, R. y Rondón, C. (1996).

Diccionario toponímico del estado Carabobo. Valencia: Clemente Editores

Hernández, R. (s.f). El origen del nombre de Venezuela es indígena, y quiere decir agua grande [Documento en línea] Recuperado de www.simonbolivar.org/Principal/bolivar/nombre_vzla. htlm [Consulta: 2011, marzo 19]

Idler, O. (1997). Toponimia carabobeña. Carabobo: Ediciones del Gobierno de Carabobo

Mata, L. (1997). Toponimia de pueblos neoespartanos. Porlamar: Fondo Editorial Fondene

Páez U., I. (2001). Comunicación, lenguaje humano y organización del código lingüístico. Valencia: Editorial Melvin

Puente, W. (s.f). Técnicas de investigación. [Documento en línea]. Recuperado de http://www.

rrppnet.com.ar/tecnicasdeinvestigacion.ht m [Consulta: 2011, mayo 22]
Rodríguez, G., Gil, J. y García, E. (1996). Metodología de la investigación cualitativa. Málaga: Aljibe

Rosenblat, A. (2004). Buenas y malas palabras. Caracas: Monte Ávila Editores Latinoamericana

Salazar, A. (1983). La toponimia venezolana en las Fuentes Cartográficas del Archivo General de Indias. Caracas: Biblioteca de la Academia Nacional de la Historia

Salazar, A. (1985). La toponimia en Venezuela. Caracas: Ediciones FACES/UCV

Salazar, A. (1990). Toponimia del Delta del Orinoco. Universidad Central de Venezuela. Cartografía Nacional. Gobernación T.F.D.A

Taylor, S. y Bogdan, R. (1987). Introducción a los métodos cualitativos de investigación. Barcelona, España: Paidós

Universidad Pedagógica Experimental Libertador, Vicerrectorado de Investigación Postgrado. (2016). Manual de Trabajos de Grado de Especialización y Maestría y Tesis Doctorales. Caracas: Autor 